\title{
CONSTRAINT QUALIFICATIONS FOR EXTENDED FARKAS'S LEMMAS AND LAGRANGIAN DUALITIES IN CONVEX INFINITE PROGRAMMING
}

\author{
D. H. FANG*, CHONG LI ${ }^{\dagger}$, AND K. F. NG $\ddagger$
}

\begin{abstract}
For an inequality system defined by a possibly infinite family of proper functions (not necessarily lower semicontinuous), we introduce some new notions of constraint qualifications in terms of the epigraphs of the conjugates of these functions. Under the new constraint qualifications, we obtain characterizations of those reverse-convex inequalities which are consequence of the constrained system, and we provide necessary and/or sufficient conditions for a stable Farkas lemma to hold. Similarly, we provide characterizations for constrained minimization problems to have the strong or strong stable Lagrangian dualities. Several known results in the conic programming problem are extended and improved.
\end{abstract}

Key words. convex inequality system, Farkas lemma, strong Lagrangian duality, conic programming

AMS(MOS) Subject Classifications. Primary, 90C34; 90C25 Secondary, 52A07; 41A29; 90C46

1. Introduction. Centered around the celebrated Farkas lemma and its extensions, we study in this paper several (old and new) constraint qualifications for optimization problems of the following type:

$$
\begin{array}{ll}
\text { Minimize } & f(x), \\
\text { s. t. } & f_{t}(x) \leq 0, t \in T, \\
& x \in C,
\end{array}
$$

where $T$ is an arbitrary (possibly infinite) index set, $C$ is a nonempty convex subset of a locally convex (Hausdorff topological vector) space $X$ and $f, f_{t}: X \rightarrow \mathbb{R} \cup\{+\infty\}, t \in T$, are proper convex functions. Throughout this paper, we assume that

$$
\emptyset \neq A:=\left\{x \in C: f_{t}(x) \leq 0, \forall t \in T\right\} .
$$

This problem has been studied extensively under various degrees of restrictions imposed on $f_{t}, t \in T$ or on the underlying space and many problems in optimization and approximation theory such as linear semi-infinite optimization and the best approximation with restricted ranges can be recast into the form (1.1), see for example $[8,16,17,23,24,33,35,36,38,40,41,42]$. Another important and classical example of (1.1) is the following so-called conic programming problem, which recently received much attention (cf. $[2,3,5,6,9,18,28,29,30,31,32]$ ),

$$
\begin{array}{ll}
\text { Minimize } & f(x), \\
\text { s. t. } & x \in C, g(x) \in-S,
\end{array}
$$

\footnotetext{
${ }^{*}$ College of Mathematics and Computer Science, Jishou University, Jishou 416000, P. R. China; Department of Mathematics, Zhejiang University, Hangzhou 310027, P. R. China, (dh_fang@jsu.edu.cn).

${ }^{\dagger}$ Corresponding author; Department of Mathematics, Zhejiang University, Hangzhou 310027, P. R. China, (cli@zju.edu.cn). This author was supported in part by the National Natural Science Foundation of China (grant 10671175, 10731060).

${ }^{\ddagger}$ Department of Mathematics, Chinese University of Hong Kong, Hong Kong, P. R. China, (kfng@math.cuhk.edu.hk). This author was supported by an Earmarked Grant (GRF) from the Research Grant Council of Hong Kong.
} 
where $X, C$ and $f$ are as in (1.1); $S$ is a closed convex cone in a locally convex space $Y, g: X \rightarrow$ $Y \cup\{+\infty\}$ is a $S$-convex function.

Observe that the most works in the literature mentioned above were done under the assumptions that for (1.1),

$C$ is closed and $f, f_{t}$, are lower semicontinuous (lsc) for all $t \in T$

and that for (1.3),

$$
C \text { is closed, } f \text { is lsc and } g \text { is } S \text {-epi-closed }
$$

(or some stronger continuity assumptions). For example, $f, f_{t}$ are continuous in $[8]$ and lsc in $[16,17$, 23], $f, g$ are continuous in [9, 18, 28, 29, 30,32], $f$ is lsc, $g$ is continuous in [31] and $g$ is $S$-epi-closed in $[5,6]$. Very recently, an optimality condition for (1.1) was established in [41] for the case when $f_{t}$ are not necessary lsc, and a Lagrangian duality result via the interiority technique for (1.3) was established in [3] without any continuity assumption on $f$ and $g$. Indeed, in the mathematical programming, many of the problems naturally involve non-convex and non-continuous functions. For example, in the DC infinite programming (see for example $[7,19,20,21,22]$ and references therein), the objective functions and constraint functions are, in general, assumed to be DC functions (such a function is, by definition, a difference of two convex functions and so can be neither convex nor lsc). Another important example is the convex composite problem which has been studied extensively by many researchers (see [14, $39,46,47,52]$ and references therein). In particular, Rockafellar [46] gave many interesting examples showing that a wide spectrum of problems can be cast in terms of convex-composite functions (which are, in general, non-convex and non-continuous). Another "nonclosed" situation naturally arises when one considers the best restricted range approximation in complex valued continuous function space $C(Q)$, which has been studied extensively (see for example [34, 35, 37, 48, 49] and reference therein), consisting of finding a best approximation to $f \in C(Q)$ from $P_{\Omega}=\left\{p \in P: p(t) \in \Omega_{t}\right.$ for all $\left.t \in Q\right\}$, where $Q$ is a compact Hausdorff space, $P$ is a finite-dimensional subspace of $C(Q)$ and $\Omega=\left\{\Omega_{t}: t \in Q\right\}$ is a system of nonempty convex set in the complex plane $\mathbb{C}$. As done in $[34,35,37,48,49]$, each $\Omega_{t}$ usually is expressed as a level set of some convex function, which is not lsc if $\Omega_{t}$ is not closed. Thus, our approach can cover the case where $\Omega_{t}$ is not necessarily closed.

Our interest for the above optimization problems in the present paper is focused on two aspects: one is about the extended Farkas lemma and the other is about the strong Lagrangian duality. Farkastype results for convex systems are fundamental in convex optimization and in other fields such as game theory, set containment problems etc. The literature on these areas is very rich (see, e.g., the survey in [26]); here let us especially mention some recent papers $[8,16,17,24,27,42]$ regarding the problem (1.1) and $[18,30,31]$ regarding (1.3). Usually for the extended Farkas lemma for (1.1), one seeks conditions ensuring the following equivalence:

$$
[f(x) \geq \alpha, \forall x \in A] \Longleftrightarrow\left[\begin{array}{l}
\exists t_{1}, \cdots, t_{m} \in T \text { and } \lambda_{t_{1}} \geq 0, \cdots, \lambda_{t_{m}} \geq 0 \\
\text { s.t. } f(x)+\sum_{i=1}^{m} \lambda_{t_{i}} f_{t_{i}}(x) \geq \alpha, \forall x \in C .
\end{array}\right]
$$

We say that the family $\left\{f, \delta_{C} ; f_{t}: t \in T\right\}$ satisfies the Farkas rule if (1.6) holds for each $\alpha \in \mathbb{R}$, and that it satisfies the stable Farkas rule if the family $\left\{f+p, \delta_{C} ; f_{t}: t \in T\right\}$ satisfies the Farkas rule for each $p \in X^{*}$. To our knowledge, not many results are known to provide a complete characterization for the Farkas rule (or the stable Farkas rule), except the works in [31] where, assuming in addition that $f$ is lsc and $g$ is continuous on $X$, a complete characterization was established for the stable 
Farkas rule for the problem (1.3), that is the characterization for the following result of Farkas-type lemma to hold: For each $x^{*} \in X^{*}$ and each $\alpha \in \mathbb{R}$,

$$
\left[-g(x) \in S \Rightarrow f(x) \geq\left\langle x^{*}, x\right\rangle+\alpha\right] \Longleftrightarrow\left(\exists \lambda \in S^{\oplus}\right)(\forall x \in X) f(x)+(\lambda \circ g)(x) \geq\left\langle x^{*}, x\right\rangle+\alpha .
$$

Their conditions are given in terms of the epigraphs of conjugate functions of $f$ and $\lambda \circ g, \lambda \in S^{\oplus}$, where

$$
S^{\oplus}:=\left\{\lambda \in Y^{*}:\langle\lambda, y\rangle \geq 0, \forall y \in S\right\} .
$$

Issues regarding the Lagrangian rules for the problems (1.1) and (1.3) are somewhat similar and most of the results in the literature are under the assumption (1.4) or (1.5), and only concern with providing sufficient conditions to ensure the (strong/strong stable ) Lagrangian duality to hold, see for example, $[5,23,31,29]$.

Constraint qualifications involving epigraphs have been studied and extensively used by Jeyakumar and his collaborators in [11, 12, 23, 28, 29, 30, 31], Botg et al. [4, 5, 6, 9, 10], Dinh et al. $[16,17,19,20,21,22]$, and Li et al. [40,41]. Our main aims in the present paper is to use these constraint qualifications (or their variations) to provide complete characterizations for the Farkas rule and the stable Farkas rule, and for the (strong/strong stable ) Lagrangian duality. In general we do not impose any topological assumption on $C$ or on $f, f_{t}$, that is $C$ is not necessarily closed and $f, f_{t}(t \in T)$ are not necessarily lsc. In particular, even in the special case when (1.4) is satisfied, our results provide improved versions of [17, Theorems 2 and 5] and that of [23, Theorem 4.1]. Moreover, applications to the problem (1.3) are given: we not only extend and improve some recent known results in $[3,5,9,18,29,31]$ but also provide new results as detailed in Section 6 .

Although most of our considerations (e.g., Theorems 4.3-4.5, 5.1 and Theorems 6.5-6.8, etc.) remain valid even if one drops the convexity assumptions of the involved sets/functions, we shall keep these convexity assumptions throughout, for the sake of simplicity in presentation. Moreover, to avoid the triviality in our study for (1.1), we always assume that

$$
\operatorname{dom} f \cap A \neq \emptyset .
$$

The paper is organized as follows. The next section contains the necessary notations and preliminary results. In Section 3, some new constraint qualifications are provided and several relationships among them are given. The characterization of the extended Farkas lemma and that of the stable extended Farkas lemma (with linear perturbations of the cost function $f$ ) are obtained in Section 4. In Section 5, we provide characterizations for minimization problems with constraints defined by inequality systems to have the strong or strong stable Lagrangian dualities. Applications to the conic programming problem are provided in Section 6 .

2. Notations and preliminary results. The notations used in this paper are standard (cf. [51]). In particular, we assume throughout the whole paper that $X$ is a real locally convex space and let $X^{*}$ denote the dual space of $X$. For $x \in X$ and $x^{*} \in X^{*}$, we write $\left\langle x^{*}, x\right\rangle$ for the value of $x^{*}$ at $x$, that is, $\left\langle x^{*}, x\right\rangle:=x^{*}(x)$. Let $Z$ be a set in $X$. The interior (resp. closure, convex hull, convex cone hull) of $Z$ is denoted by $\operatorname{int} Z$ (resp. $\operatorname{cl} Z, \operatorname{co} Z, \operatorname{cone} Z)$. The dual $X^{*}$ is endowed with the weak ${ }^{*}$-topology. Thus if $W \subseteq X^{*}$, then $\mathrm{cl} W$ denotes the weak*-closure of $W$. We shall adopt the convention that cone $Z=\{0\}$ when $Z$ is an empty set. 
The positive polar cone $Z^{\oplus}$ and the negative polar cone $Z^{\ominus}$ are defined respectively by

$$
Z^{\oplus}:=\left\{x^{*} \in X^{*}:\left\langle x^{*}, z\right\rangle \geq 0 \text { for all } z \in Z\right\}
$$

and

$$
Z^{\ominus}:=\left\{x^{*} \in X^{*}:\left\langle x^{*}, z\right\rangle \leq 0 \text { for all } z \in Z\right\} .
$$

Following [5], we use $\mathbb{R}^{(T)}$ to denote the space of real tuples $\lambda=\left(\lambda_{t}\right)_{t \in T}$ with only finitely many $\lambda_{t} \neq 0$, and let $\mathbb{R}_{+}^{(T)}$ denote the nonnegative cone in $\mathbb{R}^{(T)}$, that is

$$
\mathbb{R}_{+}^{(T)}:=\left\{\left(\lambda_{t}\right)_{t \in T} \in \mathbb{R}^{(T)}: \lambda_{t} \geq 0 \text { for each } t \in T\right\}
$$

The indicator function $\delta_{Z}$ and the support function $\sigma_{Z}$ of the nonempty set $Z$ are respectively defined by

$$
\delta_{Z}(x):=\left\{\begin{array}{cc}
0, & x \in Z \\
+\infty, & \text { otherwise }
\end{array}\right.
$$

and

$$
\sigma_{Z}\left(x^{*}\right):=\sup _{x \in Z}\left\langle x^{*}, x\right\rangle \quad \text { for each } x^{*} \in X^{*} .
$$

Let $f$ be a proper function defined on $X$. The effective domain, conjugate function and epigraph of $f$ are denoted by $\operatorname{dom} f, f^{*}$ and epi $f$ respectively; they are defined by

$$
\begin{gathered}
\operatorname{dom} f:=\{x \in X: f(x)<+\infty\}, \\
f^{*}\left(x^{*}\right):=\sup \left\{\left\langle x^{*}, x\right\rangle-f(x): x \in X\right\} \text { for each } x^{*} \in X^{*},
\end{gathered}
$$

and

$$
\text { epi } f:=\{(x, r) \in X \times \mathbb{R}: f(x) \leq r\}
$$

Then, the Young-Fenchel inequality below holds:

$$
f(x)+f^{*}\left(x^{*}\right) \geq\left\langle x^{*}, x\right\rangle \text { for each pair }\left(x, x^{*}\right) \in X \times X^{*} .
$$

It is well known and easy to verify that epi $f^{*}$ is weak*-closed. In particular,

$$
\text { epi } \delta_{Z}^{*}=\operatorname{epi} \sigma_{Z}=Z^{\ominus} \times \mathbb{R}_{+} .
$$

The closure of $f$ is denoted by $\operatorname{cl} f$, which is defined by

$$
\text { epi }(\operatorname{cl} f)=\operatorname{cl}(\operatorname{epi} f)
$$

Then (cf. [51, Theorems 2.3.1]),

$$
f^{*}=(\operatorname{cl} f)^{*}
$$

By [51, Theorem 2.3.4], if $\mathrm{cl} f$ is proper and convex, then the following equality holds:

$$
f^{* *}=\operatorname{cl} f .
$$


Furthermore, if $f, h$ are proper functions, then

$$
\text { epi } f^{*}+\operatorname{epi} h^{*} \subseteq \operatorname{epi}(f+h)^{*}
$$

and

$$
f \leq h \Longrightarrow f^{*} \geq h^{*} \Longleftrightarrow \operatorname{epi} f^{*} \subseteq \operatorname{epi} h^{*} .
$$

Finally, we introduce the definition of infimal convolution functions. Given two proper functions $f, h: X \rightarrow \mathbb{R} \cup\{+\infty\}$, the infimal convolution of $f$ and $h$ is defined by

$$
f \square h: X \rightarrow \mathbb{R} \cup\{ \pm \infty\},(f \square h)(a)=\inf _{x \in X}\{f(x)+h(a-x)\},
$$

which is said to be exact at some $a \in X$ if there is an $x \in X$ such that $(f \square h)(a)=f(x)+h(a-x)$.

The following lemma characterizes the epigraph of the conjugate of the sum of two functions.

Lemma 2.1. Let $g, h: X \rightarrow \mathbb{R} \cup\{+\infty\}$ be proper convex functions such that $\operatorname{dom} g \cap \operatorname{dom} h \neq \emptyset$.

(i) If $g$ and $h$ are lsc, then

$$
\operatorname{epi}(g+h)^{*}=\operatorname{cl}\left(\operatorname{epi}\left(g^{*} \square h^{*}\right)\right)=\operatorname{cl}\left(\operatorname{epi} g^{*}+\text { epi } h^{*}\right) .
$$

(ii) If $g$ or $h$ is continuous at some $x_{0} \in \operatorname{dom} g \cap \operatorname{dom} h$, then

$$
(g+h)^{*}=g^{*} \square h^{*}, \quad g^{*} \square h^{*} \text { is exact at every } p \in X^{*}
$$

and

$$
\operatorname{epi}(g+h)^{*}=\operatorname{epi} g^{*}+\operatorname{epi} h^{*} \text {. }
$$

Proof. Part (i) is a simple consequence of the Rockafellar-Moreau theorem (cf. [45, 50]). For (ii), one notes (by [51, Theorem 2.8.7]) that the given assumption implies that (2.6) holds and then (2.7) follows from [10, Proposition 2.2].

3. New regularity conditions. As in [51], we use $\Lambda(X)$ to denote the class of all proper convex functions on $X$. For a convex set $Z$, we write

$$
\Lambda_{Z}(X)=\{g \in \Lambda(X): \operatorname{dom} g \cap Z \neq \emptyset\} .
$$

Note that functions in $\Lambda(X)$ are not necessarily lsc. Unless explicitly stated otherwise, let $f, T, C,\left\{f_{t}\right.$ : $t \in T\}$ and $A$ be as in section 1, namely, $T$ is an index set, $C \subseteq X$ is a convex set, $f_{t} \in \Lambda(X)$ for each $t \in T$, and $A \neq \emptyset$ is the solution set of the following system:

$$
x \in C ; f_{t}(x) \leq 0 \text { for each } t \in T .
$$

Then, the following equivalence holds:

$$
[f(x) \geq \alpha, \forall x \in A] \Longleftrightarrow(0,-\alpha) \in \operatorname{epi}\left(f+\delta_{A}\right)^{*} .
$$


Following [17], the characteristic cone $K$ of (3.1) is, by definition,

$$
K:=\text { cone }\left\{\left(\operatorname{epi} \delta_{C}^{*}\right) \cup\left(\cup_{t \in T} \text { epi } f_{t}^{*}\right)\right\} .
$$

Taking into account that epi $\delta_{C}^{*}$ is a convex cone, $K$ can be re-expressed as

$$
K:=\operatorname{epi} \delta_{C}^{*}+\text { cone }\left\{\cup_{t \in T} \text { epi } f_{t}^{*}\right\}
$$

Adapting the convention that $0 \cdot \infty=0$, we have $0 \cdot h=0$ for all proper function $h$. Therefore, noting $(2.4)$

$$
\begin{aligned}
\text { epi } f^{*}+K & \subseteq \text { epi } f^{*}+\operatorname{epi} \delta_{C}^{*}+\cup_{\lambda \in \mathbb{R}_{+}^{(T)}} \operatorname{epi}\left(\sum_{t \in T} \lambda_{t} f_{t}\right)^{*} \\
& \subseteq \text { epi } f^{*}+\cup_{\lambda \in \mathbb{R}_{+}^{(T)}} \operatorname{epi}\left(\delta_{C}+\sum_{t \in T} \lambda_{t} f_{t}\right)^{*} \\
& \subseteq \cup_{\lambda \in \mathbb{R}_{+}^{(T)}} \operatorname{epi}\left(f+\delta_{C}+\sum_{t \in T} \lambda_{t} f_{t}\right)^{*} \\
& \subseteq \operatorname{epi}\left(f+\delta_{A}\right)^{*}
\end{aligned}
$$

where the last inclusion holds because of (2.5) and the fact that $f+\delta_{C}+\sum_{t \in T} \lambda_{t} f_{t} \leq f+\delta_{A}$ for each $\lambda=\left(\lambda_{t}\right)_{t \in T} \in \mathbb{R}_{+}^{(T)}$.

In the case when $f=0,(3.5)$ entails that

$$
K \subseteq \cup_{\lambda \in \mathbb{R}_{+}^{(T)}} \operatorname{epi}\left(\delta_{C}+\sum_{t \in T} \lambda_{t} f_{t}\right)^{*} \subseteq \operatorname{epi} \delta_{A}^{*}
$$

Especially, if the assumption (1.4) holds, it is known (cf. [17])

$$
\operatorname{cl} K=\operatorname{epi} \delta_{A}^{*}
$$

Throughout the remainder, we assume that (1.9) holds, namely, $f \in \Lambda_{A}(X)$. Then $f+\delta_{A}$ is proper. Therefore one can apply Lemma 2.1(i) and (3.5) to conclude easily that

$$
\operatorname{epi}\left(f+\delta_{A}\right)^{*}=\operatorname{cl}\left(\operatorname{epi} f^{*}+\operatorname{cl} K\right)=\operatorname{cl}\left(\operatorname{epi} f^{*}+K\right) \subseteq \operatorname{cl}\left(\cup_{\lambda \in \mathbb{R}_{+}^{(T)}} \operatorname{epi}\left(f+\delta_{C}+\sum_{t \in T} \lambda_{t} f_{t}\right)^{*}\right) .
$$

Regarding some possible reversed inclusions in (3.5), we introduce the following definition.

Definition 3.1. The family $\left\{\delta_{C} ; f_{t}: t \in T\right\}$ is said to have

(a) the conical EHP relative to $f$ (denote by conical $(E H P)_{f}$ ) if

$$
\text { epi }\left(f+\delta_{A}\right)^{*}=\operatorname{epi} f^{*}+\operatorname{epi} \delta_{C}^{*}+\operatorname{cone}\left(\cup_{t \in T} \text { epi } f_{t}^{*}\right)\left(=\text { epi } f^{*}+K\right)
$$

(b) the conical WEHP relative to $f$ (denote by conical $\left.(W E H P)_{f}\right)$ if

$$
\operatorname{epi}\left(f+\delta_{A}\right)^{*}=\cup_{\lambda \in \mathbb{R}_{+}^{(T)}} \text { epi }\left(f+\delta_{C}+\sum_{t \in T} \lambda_{t} f_{t}\right)^{*} \text {. }
$$

REMARK 3.1. (a) By (3.5), we see that (3.9) and (3.10) in Definition 3.1 can be equivalently replaced by

$$
\text { epi }\left(f+\delta_{A}\right)^{*} \subseteq \text { epi } f^{*}+\text { epi } \delta_{C}^{*}+\text { cone }\left(\cup_{t \in T} \text { epi } f_{t}^{*}\right),
$$


and

$$
\operatorname{epi}\left(f+\delta_{A}\right)^{*} \subseteq \cup_{\lambda \in \mathbb{R}_{+}^{(T)}} \operatorname{epi}\left(f+\delta_{C}+\sum_{t \in T} \lambda_{t} f_{t}\right)^{*}
$$

(b) In the case when $f=0$, the conical $(E H P)_{f}$ reduces to the conical EHP (see [40]), that is,

$$
\operatorname{epi} \delta_{A}^{*}=\operatorname{epi} \delta_{C}^{*}+\text { cone }\left(\cup_{t \in T} \text { epi } f_{t}^{*}\right)(=K) \text {; }
$$

equivalently,

$$
\text { epi } \delta_{A}^{*} \subseteq K
$$

(c) As the terminologies suggested,

$$
\text { the conical }(E H P)_{f} \Longrightarrow \text { the conical }(W E H P)_{f}
$$

$(\operatorname{see}(3.5))$

Extending the definition given in $[13,17,19,20,21,22]$, we say that condition $(C C)$ holds if

$$
\text { epi } f^{*}+\operatorname{cl} K \text { is weak*-closed }
$$

(regardless (1.4)) holds or not).

To establish the relationship between conditions $(E H P)_{f}$ and $(C C)$, we consider (in the spirit of the condition introduced in [25, Theorem 13]) the following condition:

$$
\operatorname{cl}\left(f+\delta_{A}\right)=\operatorname{cl} f+\delta_{A^{\mathrm{cl}}},
$$

where $A^{\mathrm{cl}}$ denotes the set defined by

$$
A^{\mathrm{cl}}:=\left\{x \in \operatorname{cl} C: \operatorname{cl} f_{t}(x) \leq 0 \text { for each } t \in T\right\} .
$$

Note that $\operatorname{cl}\left(f+\delta_{A}\right) \geq \operatorname{cl} f+\delta_{A^{\text {cl }}}$ holds trivially and (3.14) is equivalent to:

$$
\operatorname{cl}\left(f+\delta_{A}\right) \leq \operatorname{cl} f+\delta_{A^{\mathrm{cl}}} .
$$

Lemma 3.2. Assume that $\mathrm{cl} f, \mathrm{cl} f_{t}$ and $\mathrm{cl}\left(f+\delta_{A}\right)$ are proper. The following equivalences hold:

$$
(3.14) \Longleftrightarrow \operatorname{epi}\left(f+\delta_{A}\right)^{*}=\operatorname{cl}\left(\operatorname{epi} f^{*}+K\right) \Longleftrightarrow \operatorname{epi}\left(f+\delta_{A}\right)^{*} \subseteq \operatorname{cl}\left(\text { epi } f^{*}+K\right) \text {. }
$$

Proof. By (2.3) and (2.5), the following equivalences hold:

$$
(3.14) \Longleftrightarrow\left(f+\delta_{A}\right)^{*}=\left(\operatorname{cl} f+\delta_{A^{\mathrm{cl}}}\right)^{*} \Longleftrightarrow \operatorname{epi}\left(f+\delta_{A}\right)^{*}=\operatorname{epi}\left(\operatorname{cl} f+\delta_{A^{\mathrm{cl}}}\right)^{*} .
$$

By (3.15) and (3.7) (applied to $\operatorname{cl} C$ and $\left\{\mathrm{cl} f_{t}\right\}$ in place of $C$ and $\left\{f_{t}\right\}$ ),

$$
\text { epi } \delta_{A^{\mathrm{cl}}}^{*}=\operatorname{cl}\left(\operatorname{cone}\left(\text { epi } \delta_{\mathrm{cl} C}^{*}+\cup_{t \in T} \text { epi }\left(\operatorname{cl} f_{t}\right)^{*}\right)\right)=\operatorname{cl}\left(\operatorname{cone}\left(\text { epi } \delta_{C}^{*}+\cup_{t \in T} \text { epi } f_{t}^{*}\right)\right)=\operatorname{cl} K \text {. }
$$


Since $\mathrm{cl} f$ and $\delta_{A^{\mathrm{cl}}}$ are proper lsc functions, it follows from Lemma 2.1(i) that

$$
\begin{aligned}
\operatorname{epi}\left(\operatorname{cl} f+\delta_{A^{\mathrm{cl}}}\right)^{*} & =\operatorname{cl}\left(\operatorname{epi}(\operatorname{cl} f)^{*}+\operatorname{epi}_{A^{\mathrm{cl}}}^{*}\right) \\
& =\operatorname{cl}\left(\operatorname{epi} f^{*}+\operatorname{epi} \delta_{A^{\mathrm{cl}}}^{*}\right) \\
& =\operatorname{cl}\left(\operatorname{epi} f^{*}+\operatorname{cl} K\right) \\
& =\operatorname{cl}\left(\operatorname{epi} f^{*}+K\right) .
\end{aligned}
$$

This together with (3.18) proves the first equivalence in (3.17). The second equivalence in (3.17) is immediate from (3.5) and the fact that epi $\left(f+\delta_{A}\right)^{*}$ is weak ${ }^{*}$-closed.

The following proposition is direct from Lemma 3.2.

Proposition 3.3. Assume that $\mathrm{cl} f, \mathrm{cl} f_{t}$ and $\mathrm{cl}\left(f+\delta_{A}\right)$ are proper. The following equivalence holds:

$$
(E H P)_{f} \Longleftrightarrow\left[(3.14) \& \text { epi } f^{*}+K \text { is weak } k^{*} \text {-closed }\right] .
$$

Moreover, if $K$ is weak $k^{*}$-closed, then

$$
(E H P)_{f} \Longleftrightarrow[(3.14) \&(C C)]
$$

Since (3.14) is automatically satisfied and $\operatorname{cl} f, \operatorname{cl} f_{t}, \operatorname{cl}\left(f+\delta_{A}\right)$ are proper if (1.4) is assumed, we arrive at:

Corollary 3.4. Assume (1.4). The following equivalence holds:

$$
(E H P)_{f} \Longleftrightarrow \text { epi } f^{*}+K \text { is weak }{ }^{*} \text {-closed. }
$$

Moreover, if $K$ is weak* $k^{*}$ closed, then

$$
(E H P)_{f} \Longleftrightarrow(C C)
$$

The following proposition shows that, in the case when (1.4) holds, the notion of the conical $(W E H P)_{f}$ is equivalent to the closedness of the union set on the right-hand side of (3.10).

Proposition 3.5. Assume (1.4). Then the family $\left\{\delta_{C} ; f_{t}: t \in T\right\}$ has the conical $(W E H P)_{f}$ if and only if $\cup_{\lambda \in \mathbb{R}_{+}^{(T)}} \operatorname{epi}\left(f+\delta_{C}+\sum_{t \in T} \lambda_{t} f_{t}\right)^{*}$ is weak*-closed.

Proof. By the given assumption, Lemma 2.1 and (3.7), we have

$$
\operatorname{epi}\left(f+\delta_{A}\right)^{*}=\operatorname{cl}\left(\operatorname{epi} f^{*}+\operatorname{epi} \delta_{A}^{*}\right)=\operatorname{cl}\left(\operatorname{epi} f^{*}+\operatorname{cl} K\right)=\operatorname{cl}\left(\operatorname{epi} f^{*}+K\right),
$$

and, by (3.5), it holds that

$$
\text { epi } f^{*}+K \subseteq \cup_{\lambda \in \mathbb{R}_{+}^{(T)}} \operatorname{epi}\left(f+\delta_{C}+\sum_{t \in T} \lambda_{t} f_{t}\right)^{*} \subseteq \operatorname{epi}\left(f+\delta_{A}\right)^{*} .
$$

Thus

$$
\operatorname{epi}\left(f+\delta_{A}\right)^{*}=\operatorname{cl}\left(\cup_{\lambda \in \mathbb{R}_{+}^{(T)}} \operatorname{epi}\left(f+\delta_{C}+\sum_{t \in T} \lambda_{t} f_{t}\right)^{*}\right)
$$

and the result is clear. 
4. Extended Farkas Lemmas. Throughout this and the next sections, the notations $f, C$, $\left\{f_{t}: t \in T\right\}, A$ and $K$ are as explained at the begining of section 3 . Let $\alpha \in \mathbb{R}$. We study the solvability issue for the system

$$
x \in C, f_{t}(x) \leq 0(t \in T), f(x)<\alpha .
$$

Of course the insolvability of (4.1) is equivalent to saying that $f(x) \geq \alpha$ is a consequence of the system

$$
x \in C, f_{t}(x) \leq 0(t \in T),
$$

that is, the insolvability of (4.1) is equivalent to

$$
f(x) \geq \alpha \text { for each } x \in A \text {. }
$$

Replacing $f$ by all of its linear perturbed functions, we shall also consider the following condition

$$
f(x)-\langle p, x\rangle \geq \alpha \quad \text { for each } p \in X^{*} \text { and } x \in A .
$$

The following lemma provides a sufficient condition ensuring (4.3) to hold.

LEMMA 4.1. If $(0,-\alpha) \in \operatorname{epi} f^{*}+K$, then

$$
(0,-\alpha) \in \operatorname{epi}\left(f+\delta_{A}\right)^{*} \quad \text { and } \quad f(x) \geq \alpha \text { for each } x \in A .
$$

Proof. These two assertions follow from (3.5) and (3.2) respectively.

Proposition 4.2. The following statements are equivalent:

(i) For each $\alpha \in \mathbb{R}$,

$$
[f(x) \geq \alpha, \forall x \in A] \Longleftrightarrow(0,-\alpha) \in \text { epi } f^{*}+K
$$

(ii)

$$
\operatorname{epi}\left(f+\delta_{A}\right)^{*} \cap(\{0\} \times \mathbb{R})=\left(\operatorname{epi} f^{*}+K\right) \cap(\{0\} \times \mathbb{R}) .
$$

Proof. By (3.5), (i) is equivalent to

$$
(0,-\alpha) \in \operatorname{epi}\left(f+\delta_{A}\right)^{*} \Longleftrightarrow(0,-\alpha) \in \text { epi } f^{*}+K \quad \text { for each } \alpha \in \mathbb{R} .
$$

Since $(4.8) \Longleftrightarrow$ (ii) as easy to see, we then have (i) $\Longleftrightarrow$ (ii).

The following result provides a characterization of the conical $(E H P)_{f}$.

THEOREM 4.3. The following statements are equivalent:

(i) The family $\left\{\delta_{C} ; f_{t}: t \in T\right\}$ has the conical $(E H P)_{f}$.

(ii) For each $p \in X^{*}$,

$$
\operatorname{epi}\left(f-p+\delta_{A}\right)^{*} \cap(\{0\} \times \mathbb{R})=\left(\operatorname{epi}(f-p)^{*}+K\right) \cap(\{0\} \times \mathbb{R}) .
$$


(iii) For each $p \in X^{*}$ and each $\alpha \in \mathbb{R}$,

$$
[f(x) \geq\langle p, x\rangle+\alpha, \forall x \in A] \Longleftrightarrow(p,-\alpha) \in \text { epi } f^{*}+K .
$$

Proof. Because

$$
\text { epi } p^{*}=(p, 0)+\{0\} \times[0,+\infty) \text { and epi } g^{*}+\{0\} \times[0,+\infty)=\text { epi } g^{*}
$$

for any proper function $g$, we have that epi $(f-p)^{*}=$ epi $f^{*}+(-p, 0)$ and hence that, for each $p \in X^{*}$,

$$
(p,-\alpha) \in \operatorname{epi} f^{*}+K \Longleftrightarrow(0,-\alpha) \in \operatorname{epi}(f-p)^{*}+K
$$

Thus the equivalence of (ii) and (iii) follows from Proposition 4.2 (applied to $f-p$ ).

To prove $(\mathrm{i}) \Longleftrightarrow(\mathrm{ii})$, consider an arbitrary $p \in X^{*}$. By Lemma 2.1(ii), one has that

$$
\operatorname{epi}\left(f-p+\delta_{A}\right)^{*}=\operatorname{epi}\left(f+\delta_{A}\right)^{*}+\operatorname{epi}(-p)^{*}=\operatorname{epi}\left(f+\delta_{A}\right)^{*}+(-p, 0) .
$$

Hence

$$
\operatorname{epi}\left(f-p+\delta_{A}\right)^{*} \cap(\{0\} \times \mathbb{R})=\operatorname{epi}\left(f+\delta_{A}\right)^{*} \cap(\{p\} \times \mathbb{R})+(-p, 0) \text {. }
$$

Similarly,

$$
\left(\operatorname{epi}(f-p)^{*}+K\right) \cap(\{0\} \times \mathbb{R})=\left(\operatorname{epi} f^{*}+K\right) \cap(\{p\} \times \mathbb{R})+(-p, 0) .
$$

Therefore, (ii) holds if and only if

$$
\text { epi }\left(f+\delta_{A}\right)^{*} \cap(\{p\} \times \mathbb{R})=\left(\text { epi } f^{*}+K\right) \cap(\{p\} \times \mathbb{R}) \quad \text { for each } p \in X^{*}
$$

which is obviously equivalent to

$$
\text { epi }\left(f+\delta_{A}\right)^{*}=\text { epi } f^{*}+K \text {. }
$$

This proves the equivalence of (i) and (ii).

Next we give a new version of Farkas Lemma. Let us say that the family $\left\{f, \delta_{C} ; f_{t}: t \in T\right\}$ satisfies the Farkas rule if, for each $\alpha \in \mathbb{R}$, it holds that

$$
[f(x) \geq \alpha, \forall x \in A] \Longleftrightarrow\left[\exists\left(\lambda_{t}\right)_{t \in T} \in \mathbb{R}_{+}^{(T)} \text { s.t. } f(x)+\sum_{t \in T} \lambda_{t} f_{t}(x) \geq \alpha, \forall x \in C\right]
$$

and the stable Farkas rule if the family $\left\{f+p, \delta_{C} ; f_{t}: t \in T\right\}$ satisfies the Farkas rule for each $p \in X^{*}$, (the implications $(\Longleftarrow)$ in (4.14) always holds as can be verified easily; likewise, the implications $(\Longleftarrow)$ in (4.15) also always holds).

THEOREM 4.4. The following statements are equivalent:

(i) The family $\left\{f, \delta_{C} ; f_{t}: t \in T\right\}$ satisfies the Farkas rule.

(ii) For each $\alpha \in \mathbb{R}$,

$$
(0,-\alpha) \in \operatorname{epi}\left(f+\delta_{A}\right)^{*} \Longleftrightarrow\left[\exists\left(\lambda_{t}\right)_{t \in T} \in \mathbb{R}_{+}^{(T)} \text { s.t. }(0,-\alpha) \in \operatorname{epi}\left(f+\delta_{C}+\sum_{t \in T} \lambda_{t} f_{t}\right)^{*}\right] .
$$


(iii)

$$
\operatorname{epi}\left(f+\delta_{A}\right)^{*} \cap(\{0\} \times \mathbb{R})=\cup_{\lambda \in \mathbb{R}_{+}^{(T)}} \operatorname{epi}\left(f+\delta_{C}+\sum_{t \in T} \lambda_{t} f_{t}\right)^{*} \cap(\{0\} \times \mathbb{R}) .
$$

Proof. It is evident that (ii) $\Longleftrightarrow$ (iii). By (3.2), the condition stated in the left-hand side of (4.14) and that of (4.15) are equivalent. The corresponding assertion regarding the right-hand side is also valid. Therefore (4.14) and (4.15) are equivalent, and so (i) $\Longleftrightarrow(\mathrm{ii})$.

Using Theorem 4.4 and a similar argument as for Theorem 4.3, we can prove the following stable Farkas lemma in terms of the conical $(W E H P)_{f}$.

THEOREM 4.5. The following statements are equivalent:

(i) The family $\left\{f, \delta_{C} ; f_{t}: t \in T\right\}$ satisfies the stable Farkas rule.

(ii) For each $p \in X^{*}$ and each $\alpha \in \mathbb{R}$,

$$
(p,-\alpha) \in \operatorname{epi}\left(f+\delta_{A}\right)^{*} \Longleftrightarrow\left[\exists\left(\lambda_{t}\right)_{t \in T} \in \mathbb{R}_{+}^{(T)} \text { s.t. }(p,-\alpha) \in \operatorname{epi}\left(f+\delta_{C}+\sum_{t \in T} \lambda_{t} f_{t}\right)^{*}\right] .
$$

(iii) For each $p \in X^{*}$,

$$
\operatorname{epi}\left(f-p+\delta_{A}\right)^{*} \cap(\{0\} \times \mathbb{R})=\cup_{\lambda \in \mathbb{R}_{+}^{(T)}} \operatorname{epi}\left(f-p+\delta_{C}+\sum_{t \in T} \lambda_{t} f_{t}\right)^{*} \cap(\{0\} \times \mathbb{R}) .
$$

(iv) The family $\left\{\delta_{C} ; f_{t}: t \in T\right\}$ satisfies the conical $(W E H P)_{f}$.

The equivalence between (i), (ii) and (iii) in Corollary 4.6 below was established in [17, Theorem 2] for the case when $p=0$ and the assumptions (1.4) and (4.19) below were assumed:

$$
(C C) \text { and } K \text { is weak*-closed. }
$$

(These assumptions imply by Corollary 3.4 that $(E H P)_{f}$ holds.)

Corollary 4.6. The family $\left\{\delta_{C} ; f_{t}: t \in T\right\}$ has the conical $(E H P)_{f}$ if and only if for each $p \in X^{*}$ and each $\alpha \in \mathbb{R}$, the following statements are equivalent:

(i) $f(x) \geq\langle p, x\rangle+\alpha$ for all $x \in A$.

(ii) $(p,-\alpha) \in$ epi $f^{*}+K$.

(iii) There exists $\left(\lambda_{t}\right)_{t \in T} \in \mathbb{R}_{+}^{(T)}$ such that $f(x)+\sum_{t \in T} \lambda_{t} f_{t}(x) \geq\langle p, x\rangle+\alpha$ for all $x \in C$.

Proof. $(\Rightarrow)$. Suppose that the family $\left\{\delta_{C} ; f_{t}: t \in T\right\}$ has the conical $(E H P)_{f}$. By Remark 3.1(c), it has the conical $(W E H P)_{f}$. Then the result follows immediately from Theorem 4.3 and Theorem 4.5 .

$(\Leftarrow)$. Suppose that for each $p \in X^{*}$ and each $\alpha \in \mathbb{R},(i) \Longleftrightarrow(i i)$. Then the result is clear by the implication (iii) $\Longrightarrow$ (i) in Theorem 4.3.

The following example shows that the conical $(E H P)_{f}$ is strictly weaker than the condition (4.19) even in the case when the assumption (1.4) holds. 
Example 4.1. Let $X=C=\mathbb{R}^{2}$ and let $T:=(0,+\infty)$. Consider closed cones $D, A_{t},(t \in T)$ in $X$ defined by $D=\left\{\left(x_{1}, x_{2}\right): x_{1} \leq 0, x_{2} \geq 0\right\}$ and $A_{t}=\left\{\left(x_{1}, x_{2}\right): x_{2} \geq t x_{1}\right\}$. Then $D=\cap_{t \in T} A_{t}$. Noting that

$$
\operatorname{epi} \delta_{Z}^{*}=Z^{\ominus} \times \mathbb{R}_{+},
$$

where $Z$ is a convex cone $Z$ in $X$, one has in particular that epi $\delta_{D}^{*}=D^{\ominus} \times \mathbb{R}_{+}$and epi $\delta_{A_{t}}^{*}=A_{t}^{\ominus} \times \mathbb{R}_{+}$. Hence the characteristic cone $K$ of the family $\left\{\delta_{C}, \delta_{A_{t}}: t \in T\right\}$ of lsc functions is given by

$$
K=\operatorname{cone}\left(\cup_{t \in T}\left(A_{t}^{\ominus} \times \mathbb{R}_{+}\right)\right)=(\{(x, y): x>0, y<0\} \cup\{(0,0)\}) \times \mathbb{R}_{+}
$$

(so (4.19) is not satisfied). It is also clear that $K \subseteq$ epi $\delta_{D}^{*}$ and so epi $\delta_{D}^{*}+K=\operatorname{epi} \delta_{D}^{*}$. Since $\delta_{D}=\delta_{D}+\delta_{A}$ (as $D=A$ ), we then see that $\left\{\delta_{C}, \delta_{A_{t}}: t \in T\right\}$ has the conical $(E H P)_{f}$, where $f:=\delta_{D}$.

Inspired by [3, Example 3.13], we give an example for which the conical $(E H P)_{f}$ holds but $f_{t}$ are not lsc.

EXAmple 4.2. Consider the classical sequence space $l^{2}$ (consisting of all square summable sequences of real numbers, under the usual inner product); let $l_{+}^{2}$ denote the positive cone in $l^{2}$ (consisting of all sequences with nonnegative coordinates). Take $T:=(0,+\infty), b_{0}:=\left(\frac{1}{n}\right)_{n \in \mathbb{N}}$ and $b_{t}:=\left(\frac{1}{1+t}, \frac{1}{2+t}, \cdots\right) \in l^{2}$ for each $t \in T$. Let $g_{t}$ denote the linear functional defined by

$$
g_{t}(x)=\left\langle b_{t}, x\right\rangle \quad \text { for all } x \in l^{2}
$$

(thus epi $g_{t}^{*}=\left\{b_{t}\right\} \times \mathbb{R}_{+}$). Let $f_{t}:=g_{t}+\delta_{C_{t}}$, where

$$
C_{t}:= \begin{cases}\left\{\left(\lambda_{n}\right)_{n \in \mathbb{N}} \in l^{2}:-t<\lambda_{n} \leq 0, \forall n \in \mathbb{N}\right\}, & t \in(0,1), \\ \left\{\left(\lambda_{n}\right)_{n \in \mathbb{N}} \in l^{2}: 0 \leq \lambda_{n}<\frac{1}{t}, \forall n \in \mathbb{N}\right\}, & t \in[1,+\infty) .\end{cases}
$$

Note that $C_{t}$ is not closed and $f_{t}$ is not lsc. Let $A_{t}:=\left\{x=\left(\lambda_{n}\right)_{n \in \mathbb{N}} \in l^{2}: f_{t}(x) \leq 0\right\}$. Then

$$
A_{t}=\left\{\begin{array}{ll}
C_{t}, & t \in(0,1) ; \\
\{0\}, & t \in[1,+\infty)
\end{array} \quad \text { and } \quad \cap_{t \in T} A_{t}=\{0\}\right.
$$

Thus, with $X=C=l^{2}$ and the above data together with $f$ defined by

$$
f(x)=\left\{\begin{array}{l}
\|x\|, \quad \text { if } x \in b_{0}-l_{+}^{2}, \\
+\infty, \quad \text { otherwise }
\end{array}\right.
$$

we see that the feasible set $A$ as defined in (1.2) is exactly $\{0\}$. Then, clearly, $f+\delta_{A}=\delta_{A}$ and so

$$
\operatorname{epi}\left(f+\delta_{A}\right)^{*}=A^{\ominus} \times \mathbb{R}_{+}=l^{2} \times \mathbb{R}_{+} .
$$

Also, since each coordinate of $b_{0}$ is strictly positive, one has $\left(b_{0}-l_{+}^{2}\right)^{\ominus}=\{0\}$ and so, by (2.1),

$$
\text { epi } \delta_{b_{0}-l_{+}^{2}}^{*}=\left(b_{0}-l_{+}^{2}\right)^{\ominus} \times \mathbb{R}_{+}=\{0\} \times \mathbb{R}_{+} .
$$

Furthermore, one has by [51, Corollary 2.4.16], that

$$
\operatorname{epi}(\|\cdot\|)^{*}=\mathbb{B}^{*} \times \mathbb{R}_{+}
$$


( where $\mathbb{B}^{*}$ denotes the unit ball in $l^{2}$ ) and it follows from Lemma 2.1 that

$$
\text { epi } f^{*}=\operatorname{epi}\left(\|\cdot\|+\delta_{b_{0}-l_{+}^{2}}\right)^{*}=\operatorname{epi}(\|\cdot\|)^{*}+\operatorname{epi} \delta_{b_{0}-l_{+}^{2}}^{*}=\mathbb{B}^{*} \times \mathbb{R}_{+} .
$$

Note further that $C_{t}^{\ominus}=\left\{\begin{array}{ll}l_{+}^{2}, & t \in(0,1), \\ -l_{+}^{2}, & t \in[1,+\infty)\end{array}\right.$ and it follows from (2.1) that

$$
\text { epi } \delta_{C_{t}}^{*}=C_{t}^{\ominus} \times \mathbb{R}_{+}= \begin{cases}l_{+}^{2} \times \mathbb{R}_{+}, & t \in(0,1) \\ -l_{+}^{2} \times \mathbb{R}_{+}, & t \in[1,+\infty)\end{cases}
$$

Thus, by Lemma 2.1, one has

$$
\text { epi } f_{t}^{*}=\operatorname{epi}\left(g_{t}+\delta_{C_{t}}\right)^{*}=\operatorname{epi} g_{t}^{*}+\operatorname{epi} \delta_{C_{t}}^{*}= \begin{cases}\left(b_{t}+l_{+}^{2}\right) \times \mathbb{R}_{+}, & t \in(0,1), \\ \left(b_{t}-l_{+}^{2}\right) \times \mathbb{R}_{+}, & t \in[1,+\infty) .\end{cases}
$$

Hence, the characteristic cone of the family $\left\{\delta_{C}, f_{t}: t \in T\right\}$ is given by

$$
K=\operatorname{cone}\left(\cup_{t \in T} \text { epi } f_{t}^{*}\right)=l^{2} \times \mathbb{R}_{+}
$$

(because $K$ contains the set $\left(b_{\frac{1}{2}}+l_{+}^{2}+b_{1}-l_{-}^{2}\right) \times \mathbb{R}_{+}$, which equals to $\left.l^{2} \times \mathbb{R}_{+}\right)$. This together with (4.21) and (4.22) implies that

$$
\operatorname{epi}\left(f+\delta_{A}\right)^{*}=l^{2} \times \mathbb{R}_{+}=\operatorname{epi} f^{*}+l^{2} \times \mathbb{R}_{+}=\text {epi } f^{*}+K .
$$

This shows that the family $\left\{\delta_{C}, f_{t}: t \in T\right\}$ has the conical $(E H P)_{f}$.

5. Strong Lagrangian dualities. Let us use $\left(P_{f}\right)$ to denote the problem (1.1). Define the Lagrangian function $L_{f}$ on $X \times \mathbb{R}_{+}^{(T)}$ by

$$
L_{f}(x, \lambda):=f(x)+\sum_{t \in T} \lambda_{t} f_{t}(x) \quad \text { for each }(x, \lambda) \in X \times \mathbb{R}_{+}^{(T)} .
$$

Then the dual problem of $\left(P_{f}\right)$ is, by definition,

$$
\begin{array}{lll}
\left(D_{f}\right) & \text { Maximize } & \inf _{x \in C} L_{f}(x, \lambda), \\
\text { s. t. } & \lambda \in \mathbb{R}_{+}^{(T)} .
\end{array}
$$

We denote by $v\left(P_{f}\right)$ and $v\left(D_{f}\right)$ the optimal objective values of $\left(P_{f}\right)$ and $\left(D_{f}\right)$, respectively. Clearly, $v\left(P_{f}\right) \geq v\left(D_{f}\right)$, that is, the weak Lagrangian duality holds between $\left(P_{f}\right)$ and $\left(D_{f}\right)$. We say that the strong Lagrangian duality between $\left(P_{f}\right)$ and $\left(D_{f}\right)$ holds if there is no duality gap (that is $v\left(P_{f}\right)=$ $v\left(D_{f}\right)$ ) and the dual problem $\left(D_{f}\right)$ has an optimal solution, and that the strong stable Lagrangian duality between $\left(P_{f}\right)$ and $\left(D_{f}\right)$ holds if, for each $p \in X^{*}$, the strong Lagrangian duality between $\left(P_{f+p}\right)$ and $\left(D_{f+p}\right)$ holds. An important problem in convex analysis is: when does the strong Lagrangian duality hold? The following result shows that the condition (4.16) provides an answer.

ThEOREM 5.1. Each of (i)-(iii) of Theorem 4.4 is a necessary and sufficient condition for the strong Lagrangian duality between $\left(P_{f}\right)$ and $\left(D_{f}\right)$ to holds.

Proof. ( $\Longrightarrow$ ). Suppose that (i) of Theorem 4.4 holds. Let $\alpha:=v\left(P_{f}\right)$. We assume that $\alpha \neq-\infty$. Then $\alpha \in \mathbb{R}$ (as $\operatorname{dom} f \cap A \neq \emptyset$ ) and $f(x) \geq \alpha$ for each $x \in A$. Thus by (4.14), there exists $\left(\bar{\lambda}_{t}\right)_{t \in T} \in \mathbb{R}_{+}^{(T)}$ such that $f(x)+\sum_{t \in T} \bar{\lambda}_{t} f_{t}(x) \geq \alpha$ for each $x \in C$. This together with the weak 
duality shows that $\left(\bar{\lambda}_{t}\right)_{t \in T}$ is an optimal solution of $\left(D_{f}\right)$ and $v\left(P_{f}\right)=v\left(D_{f}\right)$. The same is trivially true if $\alpha=-\infty$ (then take $\bar{\lambda}_{t}=0$ for all $\left.t \in T\right)$.

$(\Longleftarrow)$. Conversely, suppose that $v\left(P_{f}\right)=v\left(D_{f}\right)$ and that the problem $\left(D_{f}\right)$ has an optimal solution $\left(\bar{\lambda}_{t}\right)_{t \in T}$. Let $\alpha \in \mathbb{R}$ be such that $f(x) \geq \alpha$ for each $x \in A$. Then $v\left(P_{f}\right)=\inf _{x \in A} f(x) \geq \alpha$ and

$$
\inf _{x \in C}\left\{f(x)+\sum_{t \in T} \bar{\lambda}_{t} f_{t}(x)\right\}=v\left(D_{f}\right)=v\left(P_{f}\right) \geq \alpha .
$$

This means that (i) of Theorem 4.4 holds because the converse implication of (4.14) holds automatically as noted earlier.

By Theorem 5.1 (applied to $f-p$ for each $p \in X^{*}$ ) and Theorem 4.5, we arrive at:

TheOREM 5.2. The family $\left\{\delta_{C} ; f_{t}: t \in T\right\}$ has the conical $(W E H P)_{f}$ if and only if the strong stable Lagrangian duality between $\left(P_{f}\right)$ and $\left(D_{f}\right)$ holds.

The following corollary, which was proved in [17, Theorem 5] under the stronger assumptions (1.4) and (4.19), is a direct consequence of Theorem 5.2.

Corollary 5.3. If the family $\left\{\delta_{C} ; f_{t}: t \in T\right\}$ has the conical $(W E H P)_{f}$, then the strong Lagrangian duality between $\left(P_{f}\right)$ and $\left(D_{f}\right)$ holds.

The following theorem gives a characterization for the conical $(W E H P)_{f}$ with $f=0$.

THEOREM 5.4. The following statements are equivalent:

(i) The family $\left\{\delta_{C} ; f_{t}: t \in T\right\}$ has the conical $(W E H P)_{0}$, that is,

$$
\operatorname{epi} \delta_{A}^{*}=\cup_{\lambda \in \mathbb{R}_{+}^{(T)}} \text { epi }\left(\delta_{C}+\sum_{t \in T} \lambda_{t} f_{t}\right)^{*}
$$

(ii) If $h \in \Lambda_{A}(X)$ is such that the family $\left\{\delta_{A}\right\}$ has the conical $(E H P)_{h}$, that is,

$$
\operatorname{epi}\left(h+\delta_{A}\right)^{*}=\operatorname{epi} h^{*}+\operatorname{epi} \delta_{A}^{*},
$$

then the strong Lagrangian duality between $\left(P_{h}\right)$ and $\left(D_{h}\right)$ holds.

(iii) If $h \in \Lambda_{A}(X)$ is continuous at some point in $A$, then the strong Lagrangian duality between $\left(P_{h}\right)$ and $\left(D_{h}\right)$ holds.

(iv) If $p \in X^{*}$, then the strong Lagrangian duality between $\left(P_{p}\right)$ and $\left(D_{p}\right)$ holds.

Proof. (i) $\Rightarrow$ (ii). Suppose that (i) holds and let $h \in \Lambda_{A}(X)$ be such that (5.4) is satisfied. Then, it follows from (5.3) and (2.4) that

$$
\begin{aligned}
\operatorname{epi}\left(h+\delta_{A}\right)^{*} & =\operatorname{epi} h^{*}+\cup_{\lambda \in \mathbb{R}_{+}^{(T)}} \operatorname{epi}\left(\delta_{C}+\sum_{t \in T} \lambda_{t} f_{t}\right)^{*} \\
& =\cup_{\lambda \in \mathbb{R}_{+}^{(T)}}\left(\operatorname{epi} h^{*}+\operatorname{epi}\left(\delta_{C}+\sum_{t \in T} \lambda_{t} f_{t}\right)^{*}\right) \\
& \subseteq \cup_{\lambda \in \mathbb{R}_{+}^{(T)}} \operatorname{epi}\left(h+\delta_{C}+\sum_{t \in T} \lambda_{t} f_{t}\right)^{*} .
\end{aligned}
$$


This shows that the family $\left\{\delta_{C} ; f_{t}: t \in T\right\}$ has the conical $(W E H P)_{h}$ (cf. Remark 3.1(a)). Applying Corollary 5.3, we complete the proof of the implication (i) $\Rightarrow$ (ii).

(ii) $\Rightarrow$ (iii). Note that (5.4) is satisfied if $h \in \Lambda_{A}(X)$ is continuous at some point in $A$ (see Lemma 2.1(ii)). Thus, it is immediate that (ii) $\Rightarrow$ (iii).

(iii) $\Rightarrow$ (iv). It is trivial.

(iv) $\Rightarrow$ (i). It is a straightforward consequence of Theorem 5.2 by letting $f=0$. $\square$

Corollary 5.5. Suppose that the following condition holds:

$$
\cup_{\lambda \in \mathbb{R}_{+}^{(T)}} \operatorname{epi}\left(\delta_{C}+\sum_{t \in T} \lambda_{t} f_{t}\right)^{*}=K .
$$

Then each of (i)-(iv) of Theorem 5.4 is equivalent to the following statement:

(v) The family $\left\{\delta_{C} ; f_{t}: t \in T\right\}$ has the conical EHP, that is, epi $\delta_{A}^{*}=K$.

Proof. Suppose that (5.5) holds. By Theorem 5.4, it suffices to verify that

$$
\text { the conical } E H P \Longleftrightarrow \text { the conical }(W E H P)_{0} \text {. }
$$

By Remark 3.1(c), we need only to prove the implication " $\Longleftarrow "$. Now if the family $\left\{\delta_{C} ; f_{t}: t \in T\right\}$ has the conical $(W E H P)_{0}$, then (5.3) holds, and so (5.5) simply means epi $\delta_{A}^{*}=K$; hence the family $\left\{\delta_{C} ; f_{t}: t \in T\right\}$ has the conical $E H P$. Thus the implication "£" of (5.6) is shown.

The following corollary was given in [23, Theorem 4.1] under the additional assumption (1.4).

Corollary 5.6. Suppose that, for each $t \in T, f_{t}$ is continuous at some point of A. Then (5.5) and the conclusion of Corollary 5.5 hold.

Proof. We need only to show (5.5). By (3.4) and (3.6), it is sufficient to show that

$$
\cup_{\lambda \in \mathbb{R}_{+}^{(T)}} \operatorname{epi}\left(\delta_{C}+\sum_{t \in T} \lambda_{t} f_{t}\right)^{*} \subseteq \text { epi } \delta_{C}^{*}+\text { cone }\left\{\cup_{t \in T} \text { epi } f_{t}^{*}\right\} .
$$

To do this, let $\lambda=\left(\lambda_{t}\right)_{t \in T} \in \mathbb{R}_{+}^{(T)}$ with $\emptyset \neq J:=\left\{t \in T: \lambda_{t} \neq 0\right\}$, say $J=\left\{t_{1}, \cdots, t_{m}\right\}$. Then we have (noting that $0 \cdot h=0$ for all functions $h$ as mentioned earlier)

$$
\operatorname{epi}\left(\delta_{C}+\sum_{t \in T} \lambda_{t} f_{t}\right)^{*}=\operatorname{epi}\left(\delta_{C}+\sum_{i=1}^{m} \lambda_{t_{i}} f_{t_{i}}\right)^{*}=\operatorname{epi} \delta_{C}^{*}+\sum_{i=1}^{m} \lambda_{t_{i}} \text { epi } f_{t_{i}}^{*},
$$

where the last equality holds because one can apply Lemma 2.1(ii) iteratively as each $\lambda_{t_{i}} f_{t_{i}}$ is continuous at some point of $A$ thanks to the given assumption. Thus (5.7) follows and the proof is complete. 口

The following example presents the case when the condition (5.5) holds but there exists some $f_{t}$ which has no continuity. Hence Corollary 5.5 is a proper extension of Corollary 5.6 (and [23, Theorem $4.1])$.

Example 5.1. Let $X=\mathbb{R}^{2}$ and let $C, D$ be closed convex cones in $X$ defined respectively by

$$
C:=\left\{\left(x_{1}, x_{2}\right): x_{1} \leq 0, x_{2} \geq 0\right\} \quad \text { and } \quad D:=\left\{\left(x_{1}, x_{2}\right): x_{1} \geq 0, x_{2} \geq 0\right\} .
$$


Then

$$
C \cap D=\left\{\left(x_{1}, x_{2}\right): x_{1}=0, x_{2} \geq 0\right\} \quad \text { and } \quad(C \cap D)^{\ominus}=C^{\ominus}+D^{\ominus} .
$$

By this and (4.20), we have that $\delta_{C \cap D}^{*}=\delta_{C}^{*}+\delta_{D}^{*}$. Since $\delta_{C \cap D}=\delta_{C}+\delta_{D}$, we have then

$$
\operatorname{epi}\left(\delta_{C}+\delta_{D}\right)^{*}=\operatorname{epi} \delta_{C}^{*}+\operatorname{epi} \delta_{D}^{*}
$$

Thus (5.5) is satisfied by the family $\left\{\delta_{C}, f_{1}\right\}$, where $f_{1}=\delta_{D}$. Note that for the above family, one has $A=C \cap D$. Clearly, $f_{1}$ is not continuous at each point of $A$.

6. Applications to Conic Programming. Throughout this section, let $X, Y$ be locally convex spaces, $C \subseteq X$ be a nonempty convex set. Let $S \subseteq Y$ be a closed convex cone. Define an order on $Y$ by saying that $y \leq_{S} x$ if $y-x \in-S$. We attach a "greatest element" $+\infty \notin Y$ with respect to $\leq_{S}$ and denote $Y^{\bullet}:=Y \cup\{+\infty\}$. The following operations are defined on $Y^{\bullet}$ : for any $y \in Y$, $y+(+\infty)=(+\infty)+y=+\infty$ and $t(+\infty)=+\infty$ for any $t \geq 0$. Let $f: X \rightarrow \mathbb{R} \cup\{+\infty\}$ be a proper convex function and $g: X \rightarrow Y^{\bullet}$ be $S$-convex in the sense that for every $u, v \in X$ and every $t \in[0,1]$,

$$
g(t u+(1-t) v) \leq_{S} t g(u)+(1-t) g(v)
$$

(see $[4,5,30])$. Consider the following conic programming problem $\left(P_{f}(S)\right)$ :

$$
\begin{array}{ll}
\text { Minimize } & f(x), \\
\text { s. t. } & x \in C, g(x) \in-S .
\end{array}
$$

Problem (6.1) has been studied in [5, 6], and also studied in [9, 28, 29, 30, 31, 33, 32] for the special case when $X, Y$ are Banach spaces (or normed spaces) and $g: X \rightarrow Y$ is $S$-convex and continuous. As in $[4,5,16,41]$, we define for each $\lambda \in S^{\oplus}$,

$$
(\lambda g)(x):= \begin{cases}\langle\lambda, g(x)\rangle & \text { if } x \in \operatorname{dom} g \\ +\infty & \text { otherwise. }\end{cases}
$$

It is easy to see that $g$ is $S$-convex if and only if $(\lambda g)(\cdot): X \rightarrow \mathbb{R} \cup\{+\infty\}$ is a convex function for each $\lambda \in S^{\oplus}$. Moreover, the problem (6.1) can be viewed as an example of (1.1) by setting

$$
T=S^{\oplus} \quad \text { and } \quad g_{\lambda}=\lambda g \quad \text { for each } \lambda \in T=S^{\oplus} .
$$

As before, we use $A$ and $K$ to denote respectively the solution set and the characteristic cone of $\left(P_{f}(S)\right)$ :

$$
A:=\left\{x \in C:(\lambda g)(x) \leq 0, \forall \lambda \in S^{\oplus}\right\}=\{x \in C: g(x) \in-S\}
$$

and

$$
K:=\operatorname{epi} \delta_{C}^{*}+\operatorname{cone}\left(\cup_{\lambda \in S^{\oplus}} \operatorname{epi}(\lambda g)^{*}\right)=\operatorname{epi} \delta_{C}^{*}+\cup_{\lambda \in S^{\oplus}} \operatorname{epi}(\lambda g)^{*},
$$

where the last equality holds because $S^{\oplus}$ is a convex cone and so

$$
\text { cone }\left(\cup_{\lambda \in S^{\oplus}} \operatorname{epi}(\lambda g)^{*}\right)=\cup_{\lambda \in S^{\oplus}} \operatorname{epi}(\lambda g)^{*} \text {. }
$$

Moreover, by (6.6), the corresponding dual problem $\left(D_{f}(S)\right)$ can be expressed as

$$
\begin{array}{ll}
\text { Minimize } & \inf _{x \in C}\{f(x)+(\lambda g)(x)\}, \\
\text { s. t. } & \lambda \in S^{\oplus} .
\end{array}
$$


In this section, we always assume that

$$
A \neq \emptyset, \quad f \in \Lambda_{A}(X) \text { and } g \text { is } S \text {-convex. }
$$

The following notion of $S$-lower semicontinuity was introduced in [44] and extended in [1, 15] for functions $g: X \rightarrow Y^{\bullet}$. It was also considered in $[4,5,41]$.

Definition 6.1. Let $g: X \rightarrow Y^{\bullet}$ be a $S$-convex function. The function $g$ is said to be

(a) S-lower semicontinuous if for each $x_{0} \in X$, each neighborhood $V$ of zero in $Y$ and any $b \in Y$ with $b \leq_{S} g\left(x_{0}\right)$, there exists a neighborhood $U$ of zero in $X$ such that

$$
g\left(x_{0}+U\right) \subseteq b+V+S \cup\{+\infty\}
$$

(b) S-epi-closed if $\operatorname{epi}_{S}(g)$ is closed, where

$$
\operatorname{epi}_{S}(g):=\{(x, y) \in X \times Y: y \in g(x)+S\} .
$$

It is known (cf. [43]) that $S$-lower semicontinuity implies that $\lambda g$ is lsc for each $\lambda \in S^{\oplus}$, which implies in turn that $g$ is $S$-epi-closed. Furthermore, if $g$ is $S$-epi-closed and $C$ is closed, then $A$ is closed and hence $\delta_{A}$ is lsc

The following lemma is taken from [5, Lemma 1].

Lemma 6.2. Suppose that $C$ is closed and $g$ is $S$-epi-closed. Then

$$
\text { epi } \delta_{A}^{*}=\operatorname{cl}\left(\operatorname{epi} \delta_{C}^{*}+\cup_{\lambda \in S^{\oplus}} \operatorname{epi}(\lambda g)^{*}\right)=\operatorname{cl} K \text {. }
$$

Generalizing the corresponding notions in $[5,6]$ to suit our present noncontinuous situation we make the following definitions (thanks to (6.5)). It is easy to see that the notions $C_{1}(f, A)$ and $C(f, A)$ in the following definition coincide with corresponding ones in $[5,6]$ (where they assumed that $(1.5)$ holds).

Definition 6.3. We say that $\left\{\delta_{C} ; g\right\}$ satisfies $(6.5))$,

(a) the condition $C_{1}(f, A)$ if the family $\left\{\delta_{C} ; \lambda g: \lambda \in S^{\oplus}\right\}$ has the conical $(E H P)_{f}$, that is (by

$$
\operatorname{epi}\left(f+\delta_{A}\right)^{*}=\operatorname{epi} f^{*}+\operatorname{epi} \delta_{C}^{*}+\cup_{\lambda \in S^{\oplus}} \operatorname{epi}(\lambda g)^{*}\left(=\text { epi } f^{*}+K\right)
$$

(b) the condition $C(f, A)$ if the family $\left\{\delta_{C} ; \lambda g: \lambda \in S^{\oplus}\right\}$ has the conical $(W E H P)_{f}$, that is,

$$
\text { epi }\left(f+\delta_{A}\right)^{*}=\cup_{\lambda \in S} \oplus \text { epi }\left(f+\delta_{C}+(\lambda g)\right)^{*} ;
$$

(c) the Farkas rule (resp. the stable Farkas rule) with respect to $f$ if the family $\left\{f, \delta_{C} ; \lambda g: \lambda \in S^{\oplus}\right\}$ satisfies the Farkas rule (resp. the stable Farkas rule).

REMARK 6.1. By Remark 3.1(a), we see that (6.10) and (6.11) in Definition 6.3 are respectively equivalent to

$$
\text { epi }\left(f+\delta_{A}\right)^{*} \subseteq \text { epi } f^{*}+\text { epi } \delta_{C}^{*}+\cup_{\lambda \in S} \oplus \operatorname{epi}(\lambda g)^{*},
$$


and

$$
\operatorname{epi}\left(f+\delta_{A}\right)^{*} \subseteq \cup_{\lambda \in S^{\oplus}} \text { epi }\left(f+\delta_{C}+(\lambda g)\right)^{*} .
$$

Let cont $h$ denote the set of all points at which $h$ is continuous, that is,

$$
\text { cont } h=\{x \in X: h \text { is continuous at } x\} .
$$

The following proposition (except the last assertion) is due to Bot et al. (see [5, 6] where the first equality of (6.14) together with (i) and (ii) were established while the second equality in (6.14) is an easy consequence of the first).

Proposition 6.4. Assume (1.5). Then

$$
\operatorname{epi}\left(f+\delta_{A}\right)^{*}=\mathrm{cl}\left(\mathrm{epi} f^{*}+K\right)=\operatorname{cl}\left(\cup_{\lambda \in S^{\oplus}} \text { epi }\left(f+\delta_{C}+\lambda g\right)^{*}\right) \text {. }
$$

Consequently,

(i) the family $\left\{\delta_{C} ; g\right\}$ satisfies $C_{1}(f, A)$ if and only if epi $f^{*}+K$ is weak ${ }^{*}$-closed;

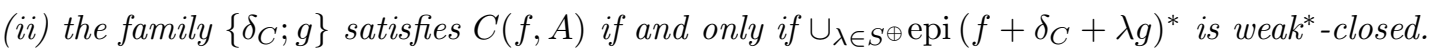
Moreover, if cont $f \cap A \cap \operatorname{int} C \neq \emptyset$, then the following equivalence holds for the family $\left\{\delta_{C} ; g\right\}$ :

$$
C_{1}(f, A) \Longleftrightarrow C(f, A) \text {. }
$$

Proof. By [5, 6], we only need to prove the last assertion. Suppose that

$$
\operatorname{cont} f \cap A \cap \operatorname{int} C \neq \emptyset .
$$

It suffices to show that the set on the right-hand side in (6.10) and that in (6.11) are the same. But this is immediate from Lemma 2.1(ii) and (6.16):

$$
\text { epi }\left(f+\delta_{C}+(\lambda g)\right)^{*}=\text { epi } f^{*}+\operatorname{epi}\left(\delta_{C}+(\lambda g)\right)^{*}=\operatorname{epi} f^{*}+\operatorname{epi} \delta_{C}^{*}+\operatorname{epi}(\lambda g)^{*} \quad \text { for each } \lambda \in S^{\oplus} .
$$

The proof is completed.

By Theorems 4.3, 4.4, 4.5, 5.1, 5.2, 5.4 and Corollary 5.5 (applied to $\left\{\delta_{C} ; \lambda g: \lambda \in S^{\oplus}\right\}$, and thanks to (6.5) and the fact that $S^{\oplus}$ is a convex cone), we have the following Theorems 6.5-6.8 and Corollary 6.9. The equivalence (ii) $\Longleftrightarrow$ (iii) in Theorems 6.7 and 6.8 was given in [5, Theorems 1 and 2] while $(\mathrm{i}) \Longleftrightarrow(\mathrm{v})$ in Theorem 6.8 was given in [5, Corollary 1] under stronger assumptions (assumption $(1.5))$.

THEOREM 6.5. The following statements are equivalent:

(i) For each $p \in X^{*}$ and each $\alpha \in \mathbb{R}$,

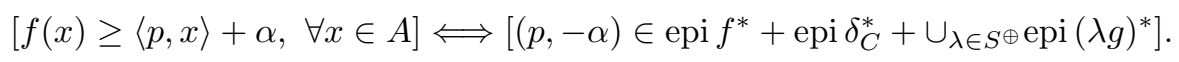

(ii) The family $\left\{\delta_{C} ; g\right\}$ satisfies the condition $C_{1}(f, A)$.

THEOREM 6.6. The following statements are equivalent: 
(i) The family $\left\{\delta_{C} ; g\right\}$ satisfies the Farkas rule with respect to $f$.

(ii) The strong Lagrangian duality between $\left(P_{f}(S)\right)$ and $\left(D_{f}(S)\right)$ holds.

(iii)

$$
\operatorname{epi}\left(f+\delta_{A}\right)^{*} \cap(\{0\} \times \mathbb{R})=\left(\cup_{\lambda \in S \oplus} \operatorname{epi}\left(f+\delta_{C}+(\lambda g)\right)^{*}\right) \cap(\{0\} \times \mathbb{R}) .
$$

THEOREM 6.7. The following statements are equivalent:

(i) The family satisfies the stable Farkas rule with respect to $f$.

(ii) The strong stable Lagrangian duality between $\left(P_{f}(S)\right)$ and $\left(D_{f}(S)\right)$ holds.

(iii) The family $\left\{\delta_{C} ; g\right\}$ satisfies the condition $C(f, A)$.

THEOREM 6.8. The following statements are equivalent:

(i) The family $\left\{\delta_{C} ; g\right\}$ satisfies the condition $C(0, A)$.

(ii) If $h \in \Lambda_{A}(X)$ and epi $\left(h+\delta_{A}\right)^{*}=$ epi $h^{*}+$ epi $\delta_{A}^{*}$, then the strong Lagrangian duality between $\left(P_{h}(S)\right)$ and $\left(D_{h}(S)\right)$ holds.

(iii) If $h \in \Lambda_{A}(X)$ is continuous at some point in $A$, then the strong Lagrangian duality between $\left(P_{h}(S)\right)$ and $\left(D_{h}(S)\right)$ holds. holds.

(iv) If $h \in \Lambda_{A}(X)$ is continuous, then the strong Lagrangian duality between $\left(P_{h}(S)\right)$ and $\left(D_{h}(S)\right)$

(v) If $p \in X^{*}$, then the strong Lagrangian duality between $\left(P_{p}(S)\right)$ and $\left(D_{p}(S)\right)$ holds.

COROLlary 6.9. Suppose that the following condition holds:

$$
\cup_{\lambda \in S \oplus}\left(\delta_{C}+(\lambda g)\right)^{*}=\operatorname{epi} \delta_{C}^{*}+\cup_{\lambda \in S^{\oplus}} \operatorname{epi}(\lambda g)^{*} .
$$

Then each of (i)-(v) of Theorem 6.8 is equivalent to the following statement:

(vi) The family $\left\{\delta_{C} ; g\right\}$ satisfies the condition $C_{1}(0, A)$.

Under the stronger assumptions ( $X, Y$ are Banach spaces, $C=X$ and $f, g$ are continuous), the implications (i) $\Longleftrightarrow$ (iii) and (ii) $\Longleftrightarrow$ (iii) in the following corollary were given respectively in Theorem 3.1 and Theorem 4.1 of [31], while the implications (iv) $\Rightarrow$ (i)\&(ii) improve [18, Theorem 2.2] by allowing general $p \in X^{*}$ rather than only $p=0$.

Corollary 6.10. Suppose that $C$ is closed, $g$ is $S$-epi-closed and that $\operatorname{cont} f \cap A \neq \emptyset$. Consider the following statements:

(i) Same as (i) in Theorem 6.7.

(ii) Same as (i) in Theorem 6.5.

(iii) epi $f^{*}+$ epi $\delta_{C}^{*}+\cup_{\lambda \in S \oplus \operatorname{epi}}(\lambda g)^{*}\left(=\operatorname{epi} f^{*}+K\right)$ is weak $k^{*}$-closed.

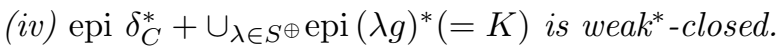

Then, (iv) $\Longrightarrow[(i)$, (ii)\&(iii)]. Moreover, (i) $\Longleftrightarrow(i i) \Longleftrightarrow$ (iii) provided that $\operatorname{cont} f \cap A \cap \operatorname{int} C \neq \emptyset$. 
Proof. (iv) $\Longrightarrow[(\mathrm{i}),(\mathrm{ii}) \&(\mathrm{iii})]$. By Lemma 6.2 and (iv), one has epi $\delta_{A}^{*}=\mathrm{cl} K=K$. Hence (iv) and Lemma 2.1(ii) (thanks to the assumption that cont $f \cap A \neq \emptyset$ ) imply that

$$
\operatorname{epi}\left(f+\delta_{A}\right)^{*}=\operatorname{epi} f^{*}+\operatorname{epi} \delta_{A}^{*}=\operatorname{epi} f^{*}+K
$$

This means that the family $\left\{\delta_{C} ; g\right\}$ satisfies $C_{1}(f, A)$ and so $C(f, A)$ (by definitions and Remark 3.1(c)). Hence (i) and (ii) hold by Theorems 6.7 and 6.5. Furthermore, (6.19) implies that epi $f^{*}+K$ is weak*-closed (because epi $\left(f+\delta_{A}\right)^{*}$ is so); hence (iii) holds.

Finally, we assume that $\operatorname{cont} f \cap A \cap \operatorname{int} C \neq \emptyset$. Then (6.15) of Proposition 6.4 holds and so the following equivalences hold for the family $\left\{\delta_{C}, g\right\}$ :

$$
C(f, A) \Longleftrightarrow C_{1}(f, A) \Longleftrightarrow \text { epi } f^{*}+K \text { is weak* }{ }^{*} \text { closed }
$$

and therefore $(\mathrm{i}) \Longleftrightarrow$ (ii) $\Longleftrightarrow$ (iii) by Theorems 6.5 and 6.7 .

The equivalence (iii) $\Longleftrightarrow$ (iv) in the following corollary was proved in [29, Theorem 3.1] under the stronger assumption that $g$ is continuous.

Corollary 6.11. Suppose that $C$ is closed, $g$ is $S$-epi-closed and that $\operatorname{cont} g \cap A \neq \emptyset$. Then the following statements are equivalent:

(i) The family $\left\{\delta_{C} ; g\right\}$ satisfies the condition $C(0, A)$.

(ii) The family $\left\{\delta_{C} ; g\right\}$ satisfies the condition $C_{1}(0, A)$.

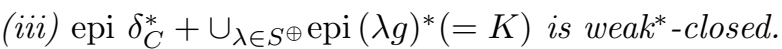

(iv) The strong Lagrangian duality between $\left(P_{h}(S)\right)$ and $\left(D_{h}(S)\right)$ holds whenever $h \in \Lambda_{A}(X)$ is continuous.

Proof. Since cont $g \cap A \neq \emptyset$, it follows that cont $(\lambda g) \cap A \neq \emptyset$ for each $\lambda \in S^{\oplus}$. Then by Definition 6.3 and Corollary 5.6 (applied to $\left\{C, \lambda g: \lambda \in S^{\oplus}\right\}$ in place of $\left\{C, f_{t}: t \in T\right\}$ ), we have (i) $\Longleftrightarrow$ (ii). Further, by Theorem 6.8, we have (i) $\Longleftrightarrow(\mathrm{iv})$. Finally, by Proposition 6.4(i) and thanks to the given assumptions, (ii) $\Longleftrightarrow$ (iii).

REMARK 6.2. Let $Z$ be a convex subset of $X$. Recall (cf. [3]) that the quasi-relative interior and the quasi interior of $Z$ are defined respectively by

qri $Z:=\{x \in Z: \operatorname{cl} \operatorname{cone}(Z-x)$ is linear $\} \quad$ and $\quad$ qi $Z:=\{x \in Z: \operatorname{cl} \operatorname{cone}(Z-x)=X\}$.

Bot et al. [3] established the strong Lagrange duality between problem $\left(P_{f}(S)\right)$ and $\left(D_{f}(S)\right)$ under the following interiority condition:

$$
0 \in \mathrm{qi}[(g(C)+S)-(g(C)+S)], \quad 0 \in \operatorname{qri}(g(C)+S) \text { and }(0,0) \notin \operatorname{qrico}\left(\varepsilon_{v\left(P_{f}(S)\right)} \cup\{(0,0)\}\right),
$$

where

$$
\varepsilon_{v\left(P_{f}(S)\right)}=\left\{\left(f(x)+\alpha-v\left(P_{f}(S)\right), g(x)+y\right): x \in C, \alpha \geq 0, y \in S\right\} \subseteq \mathbb{R} \times Y .
$$

Clearly, combining Theorem 6.7 and [3, Theorem 4.2], we see that the interiority condition (6.20) implies (6.18). The following example shows that the converse is not true. 
Example 6.1. Consider the real space $X=C=\mathbb{R}$. Let $S=\mathbb{R}_{+}$and let $f, g: \mathbb{R} \rightarrow \overline{\mathbb{R}}$ be defined by $f=\delta_{[0,1)}$ and $g=\delta_{(-1,0]}$ respectively. Then $A=\{x \in C: g(x) \in-S\}=(-1,0]$ and $f+\delta_{A}=f+g$. Noting $\lambda g=g$ for all $\lambda>0$, it follows that

$$
\text { epi }\left(f+\delta_{A}\right)^{*}=\operatorname{epi}(f+g)^{*} \subseteq \cup_{\lambda \in S^{\oplus}} \operatorname{epi}(f+\lambda g)^{*} ;
$$

hence $C(f, A)$ holds (thanks to Remark 6.1) and so does the condition (6.18). However, the above interiority condition (6.20) does not hold as $0 \notin \mathrm{qri}(g(X)+S)$ (since cl cone $g(X)+S=\mathbb{R}_{+}$is not a linear subspace of $\mathbb{R})$.

Acknowledgement. The authors are grateful to both anonymous reviewers for valuable suggestions and remarks helping to improve the quality of the paper.

\section{REFERENCES}

[1] M. Aït Mansoour, A. Metrane and M. Théra, Lower semicontinuous regularization for vector-valued mappings, J. Global Optim., 35 (2006), pp. 283-309.

[2] V. Barbu and T. Precupanu, Convexity and Optimization in Banach Spaces, Sijthoff \& Noordhoff, Alphen aan den Rijn, The Netherlands, 1978.

[3] R. I. Bot, E. R. Csetnek and G. Wanka, Regularity conditions via quasi-relative interior in convex programming, SIAM. J. Optim., 19 (2008), pp. 217-233.

[4] R. I. Boț, S. M. Grad and G. Wanka, A new constraint qualification for the formula of the subdifferential of composed convex functions in infinite dimensional spaces, Math. Nachr., 281 (2008), pp. 1088-1107

[5] R. I. Bot,, S. M. Grad and G. Wanka, On strong and total Lagrange duality for convex optimization problems, J. Math. Anal. Appl., 337 (2008), pp. 1315-1325.

[6] R. I. Bot, S. M. Grad and G. Wanka, New regularity conditions for strong and total Fenchel-Lagrange duality in infinite dimensional spaces, Nonlinear Anal., 69 (2008), pp. 323-336.

[7] R. I. Bot, I. B. Hodrea and G. Wanka, Some new Farkas-type results for inequality systems with DC functions, J. Global Optim., 39 (2007), pp. 595-608.

[8] R. I. Bọt and G. Wanka, Farkas-type results with conjugate functions, SIAM J. Optim., 15 (2005), pp. 540-554.

[9] R. I. Bot and G. Wanka, An alternative formulation for a new closed cone constraint qualification, Nonlinear Anal., 64 (2006), pp. 1367-1381.

[10] R. I. Bot and G. Wanka, A weaker regularity condition for subdifferential calculus and Fenchel duality in infinite dimensional spaces, Nonlinear Anal., 64 (2006), pp. 2787-2804.

[11] R. S. Burachik and V. Jeyakumar, A simple closure condition for the normal cone intersection formula, Proc. Amer. Math. Soc., 133 (2005), pp. 1741-1748.

[12] R. S. Burachik and V. Jeyakumar, A new geometric condition for Fenchel's duality in infinite dimensional spaces, Math. Program., Ser. B, 104 (2005), pp. 229-233.

[13] R. S. Burachik and V. Jeyakumar, A dual condition for the convex subdifferential sum formula with applications, J. Convex Anal., 12 (2005), pp. 279-290.

[14] J. V. Burke and M. C. Ferris, A Gauss-Newton method for convex composite optimization, Math. Program., 71 (1995), pp. 179-194.

[15] C. Combari, M. Laghdir and L. Thibault, Sous-différentiels de foncitions convexes composées, Ann. Sci. Math. Quebec, 18 (1994), pp. 119-148.

[16] N. Dinh, M. A. Goberna and M. A. López, From linear to convex systems: consistency, Farkas lemma and applications, J. Convex Anal., 13 (2006), pp. 279-290.

[17] N. Dinh, M. A. Goberna, M. A. López and T. Q. Son, New Farkas-type constraint qualifications in convex infinite programming, ESAIM Control Optim. Calc. Var., 13 (2007), pp. 580-597.

[18] N. Dinh, V. Jeyakumar and G. M. Lee, Sequential Lagrangian conditions for convex programs with applications to semidefinite programming, J. Optim. Theory Appl., 125 (2005), pp. 85-112.

[19] N. Dinh, B. Mordukhovich and T. T. A. Nghia, Subdifferentials of value functions and optimality conditions for DC and bilevel infinite and semi-infinite programs, Research Report 34 (2008); to appear in Math. Programming. http://www.hcmiu.edu.vn/math/preprints1.htm.

[20] N. Dinh, B. Mordukhovich and T. T. A. Nghia, Qualification and optimality conditions for convex and DC programs with infinite constraints, Research Report 29 (2008); to appear in Acta Math. Vietnamica. http://www.hcmiu.edu.vn/math/preprints1.htm. 
[21] N. Dinh, T. T. A. Nghia and G. Vallet, A closedness condition and its applications to DC programs with convex constraints, to appear in Optimization, http://dx.doi.org/10.1080/02331930801951348.

[22] N. Dinh, G. Vallet and T. T. A. Nghia, Farkas-type results and duality for DC programs with convex constraints, J. Convex Anal., 15 (2008), pp. 235-262.

[23] M. A. Goberna, V. Jeyakumar and M. A. López, Necessary and sufficient conditions for solvability of systems of infinite convex inequalities, Nonlinear Anal., 68 (2008), pp. 1184-1194.

[24] M. A. Goberna and M. A. López, Linear Semi-infinite Optimization, J. Wiley, Chichester, 1998.

[25] H. Hantoute, M. A. López, C. Zălinescu, Subdifferential calculus rules in convex analysis: A unifying approach via pointwise supremum functions, SIAM J. Optim., 19 (2008), pp. 863-882.

[26] V. Jeyakumar, Farkas lemma: Generalizations, in Encyclopedia of Optimization II, C.A. Floudas and P. Pardalos Eds., Kluwer, Dordrecht (2001), pp. 87-91.

[27] V. Jeyakumar, Characterizing set containments involving infinite convex constraints and reverse-convex constraints, SIAM J. Optim., 13 (2003), pp. 947-959.

[28] V. Jeyakumar, The strong conical hull intersection property for convex programming, Math. Program., Ser. A, 106 (2006), pp. 81-92.

[29] V. Jeyakumar, Constraint qualifications characterizing Lagrangian duality in convex optimization, J. Optim. Theory Appl., 136 (2008), pp. 31-41.

[30] V. Jeyakumar, N. Dinh and G. M. Lee, New sequential Lagrange multiplier conditions characterizing optimality without constraint qualification for convex programs, SIAM J. Optim., 14 (2003), pp. 534-547.

[31] V. Jeyakumar and G. M. Lee, Complete characterization of stable Farkas' lemma and cone-convex programming duality, Math. Program., Ser. A, 114 (2008), pp. 335-347.

[32] V. Jeyakumar, G. M. Lee and N. Dinh, Lagrange multiplier conditions characterizing the optimal solution sets of cone-constrained convex programs, J. Optim. Theory Appl., 123 (2004), pp. 83-103.

[33] V. Jeyakumar and H. Mohebi, Limiting $\epsilon$-subgradient characterizations of constrained best approximation, J. Approx. Theory, 135 (2005), pp. 145-159.

[34] C. Li, On best uniform restricted range approximation in complex-valued continuous function spaces, J. Approx. Theory, 120 (2003) pp. 71-84.

[35] C. Li and K. F. Ng, Constraint qualification, the strong CHIP and best approximation with convex constraints in Banach spaces, SIAM J. Optim., 14 (2003), pp. 584-607.

[36] C. Li and K. F. Ng, On constraint qualification for infinite system of convex inequalities in a Banach space, SIAM J. Optim., 15 (2005), pp. 488-512.

[37] C. Li and K. F. Ng, On best restricted range approximation in continuous complex-valued function spaces, J Approx. Theory, 136 (2005), pp. 159-181.

[38] C. Li and K. F. Ng, Strong CHIP for infinite system of closed convex sets in normed linear spaces, SIAM J. Optim., 16 (2005), pp. 311-340.

[39] C. Li and K. F. Ng, Majorizing functions and convergence of the Gauss-Newton method for convex composite optimization, SIAM J. Optim., 18 (2007), pp. 613-642.

[40] C. Li, K. F. Ng and T. K. Pong, The SECQ, linear regularity and the strong CHIP for infinite system of closed convex sets in normed linear spaces, SIAM J. Optim., 18 (2007), pp. 643-665.

[41] C. Li, K. F. Ng and T. K. Pong, Constraint qualifications for convex inequality systems with applications in constrained optimization, SIAM J. Optim., 19 (2008), pp. 163-187.

[42] W. Li, C. Nahak and I. Singer, Constraint qualifications for semi-infinite systems of convex inequalities, SIAM J. Optim., 11 (2000), pp. 31-52.

[43] D. T. Luc, Theorey of Vector Optimization, Spring-Verlag, Berlin, 1989.

[44] J. P. Penot and M. Théra, Semi-continuous mappings in general topology, Arch. Math., 38 (1982), pp. 158-166.

[45] R. T. Rockafellar, Extension of Fenchel's duality theorem for convex functions, Duke Math. J., 33 (1966), pp. 81-89.

[46] R. T. Rockafellar, First and second-order epidiffrentiability in nonlinear programming, Trans. Amer. Math. Soc. 307 (1988), pp.75-108.

[47] R. T. Rockafellar and R. J. B. Wets, Variational Analysis, Springer, Heidelberg, 1998.

[48] G. S. Smirnov and R. G. Smirnov, Best uniform restricted range approximation of complex-valued functions, C. R. Math. Acad. Sci. Canada, 19 (1997), pp. 58-63.

[49] G. S. Smirnov and R. G. Smirnov, Best uniform approximation of complex-valued functions by generalized polynomialsha ving restricted range, J. Approx. Theory, 100 (1999), pp. 284-303.

[50] T. Sträberg, The operation of infimal convolution, Diss. Math., 352 (1996), pp. 1-58.

[51] C. Zălinescu, Convex Analysis in General Vector Spaces, World Scientific, New Jersey, 2002.

[52] X. Y. Zheng and K. F. Ng, Strong KKT conditions and weak sharp solutions in convex-composite optimization, to appear in Math. Programming. 